\title{
Der Libanonkonflikt 2006 und das Völkerrecht
}

\author{
Von Gerd Seidel, Berlin
}

Der am 13. Juli 2006 begonnene und durch einen vom UN-Sicherheitsrat initiierten Waffenstillstand $^{1}$ am 14. August 2006 beendete Libanonkonflikt hat in rechtlicher wie in politischer Hinsicht eine Reihe von Fragen aufgeworfen, die trotz ihrer grundsätzlichen Natur bisher nur wenig diskutiert wurden. Solche Fragen sind: Wer ist Aggressor in diesem Konflikt? Wie sind die im Verlaufe des Konflikts vorgenommenen Militäraktionen nach humanitärem Völkerrecht zu bewerten? Welche Schlussfolgerungen ergeben sich daraus für die völkerrechtliche Verantwortlichkeit der Konfliktparteien und der verantwortlichen Individuen nach Völkerstrafrecht? Welche Möglichkeiten der Konfliktlösung bieten sich bei Einbettung der Libanonfrage in den Nahostkonflikt?

\section{Vorgeschichte und Verlauf}

Die Beziehungen zwischen Libanon und Israel sind seit Jahrzehnten gespannt und von bewaffneten Auseinandersetzungen - allerdings kaum mit der libanesischen Armee geprägt. Der den ersten Nahostkrieg abschließende Waffenstillstand von 1949 ordnete an, dass im Grenzgebiet beider Staaten nur eine geringe Truppenzahl mit leichter Bewaffnung zu stationieren sei. Obwohl im Gefolge des ersten Nahostkrieges etwa 100.000 palästinensische Flüchtlinge in dem an Fläche (10.452 $\left.\mathrm{km}^{2}\right)$ und Bevölkerung (ca. 3,8 Millionen) kleinen Libanon Aufnahme fanden, kam es über knapp zwei Jahrzehnte zu keinen größeren militärischen Gefechten an der gemeinsamen Grenze. Erst die durch den "Sechstagekrieg" 1967 erneut in großem Maße ausgelöste Flüchtlingswelle führte dazu, dass später bewaffnete Kämpfer aus großen palästinensischen Flüchtlingslagern - zumeist unter der Regie der PLO - vom libanesischen Gebiet aus wiederholt blutige Anschläge in Israel verübten. Israel antwortete jeweils mit Kommandounternehmen, Bombardierungen und 1978 mit einer großflächigen militärischen Intervention. Womöglich aus Rache für das von Yasir Arafat zu verantwortende Massaker im libanesischen Damour, wo 1976 die PLO an die 500 Christen ermordet hatte und in Reaktion auf die Ermordung des christlichen Präsidenten Gemayel, verübten 1982 christliche Falangisten bei Untätigkeit des vom damaligen israelischen Verteidigungsminister und nachmaligen Ministerpräsidenten Ariel Scharon geführten israelischen Militärs ein Massaker in den Beiruter Flüchtlingslagern Sabra und Schatilla, dem 
nach libanesischen Regierungsangaben 460, nach anderen Angaben an die 2.000 Personen zum Opfer fielen.

Gleichzeitig wurde die PLO aus dem Libanon ins tunesische Exil vertrieben, und die israelische Armee besetzte den Süden Libanons. In dieser Zeit erstarkte die Hisbollah ("Partei Gottes"), deren militärischer Arm - außerhalb staatlich-libanesischer Strukturen, mit syrischer Unerstützung und iranischen Waffen - fortan im Südlibanon die israelische Besatzung bekämpfte. Ungeachtet deren von den UN offiziell bestätigter Beendigung im Jahr 2000 setzte die Hisbollah vom Libanon aus den Kampf gegen Israel fort: Die Bilanz des israelischen Außenministeriums vom Juni 2006 zu den zwischen 2000 und Juni 2006 im nördlichen Grenzgebiet registrierten etwa 38 Feuerüberfällen, Sprengstoffattentaten und Raketenangriffen auf israelischer Seite: 21 Soldaten und ein UN-Beobachter getötet, 30 verwundet, drei Soldaten entführt und ermordet (07.10.2000) sowie 6 getötete und 6 verletzte Zivilpersonen. Die Hisbollah-Führung begründete ihre Aktionen mit den andauernden Verletzungen des libanesischen Luftraumes durch israelische Kampfflugzeuge, mit der Weigerung Israels, dem Libanon Karten mit den Minenfeldern zu übergeben und libanesische Hisbollah-Angehörige aus israelischer Strafhaft zu entlassen sowie mit der Solidarität gegenüber den unter israelischer Besatzung lebenden Palästinensern.

Die Resolution 1559 (2004) des UN-Sicherheitsrats, die vom Libanon insbesondere die Entwaffnung der im Süd-Libanon agierenden Milizen sowie von allen übrigen Beteiligten die Achtung der territorialen Integrität des Libanon fordert, wurde nirgendwo umgesetzt, trotz nachdrücklicher Anmahnung noch Ende Mai 2006. ${ }^{2}$ Die Hisbollah war zu einem Waffenverzicht nicht bereit, und Israel setzte seine Inspektionsflüge über dem Süd-Libanon dementsprechend fort.

Ende Mai 2006 war die Situation eskaliert dadurch, dass der libanesische Geheimdienst den israelischen Geheimdienst u.a. für die gezielte Tötung eines Führers des Islamischen Dschihad am 26. Mai 2006 verantwortlich zu machen suchte. Damit begründete die Hisbollah ihre Raketenangriffe auf Militärbasen in Israel, die die israelische Seite jeweils mit Luftschlägen beantwortete.

Unmittelbarer Anlass für den bewaffneten Konflikt war dann am 12. Juli 2006 das abermalige Eindringen eines Hisbollah-Kommandos auf israelisches Territorium und die Entführung zweier israelischer Soldaten von dort in den Libanon, wobei weitere sechs - nach for the Secretary-General on Lebanon, 29 May 2006: "The Secretary-General continues to follow the situation closely, and calls on all parties to exercise maximum restraint and respect fully the Blue Line. He particularly urges the Government of Lebanon to make every effort to exercise its control over the use of force from its territory...". 
anderen Angaben drei - Israelis umkamen. Am folgenden Tag begann die israelische Armee ihre Offensive "Angemessener Preis" gegen die vom Libanon aus operierende Hisbollah. Dabei bombardierte die Luftwaffe etwa 1.800 Gebäude, darunter neben einzelnen Wohnhäusern auch Wohnsiedlungen mit Schulen und Moscheen, deren Nähe die Hisbollah gesucht hatte, sowohl in Grenznähe als auch in und um Beirut und nördlich davon. Um den iranisch-syrischen Waffennachschub für die Hisbollah zu unterbinden, konzentrierten sich die israelischen Artillerie- und Luftschläge auf die hierfür genutzte Infrastruktur des Libanon: 630 km Straßen, 78 Brücken, die Autobahn Damaskus-Beirut und der Beiruter Flughafen, der daraufhin geschlossen werden musste. Am 14. Juli löste die Zerstörung eines küstennahen Elektrizitätswerkes eine der bislang größten Umweltkatastrophen aus, als nach WWF-Angaben etwa 12.000 Tonnen Heizöl ins Meer flossen. Am gleichen Tag verhängte Israel eine Luft- und Seeblockade über bzw. um den Libanon. Dies erschwerte internationale humanitäre Hilfsaktionen und behinderte auch die Eindämmung des Ölteppichs auf dem Meer. Von den Israelis vorübergehend eingeräumte "humanitäre Korridore" konnten nur begrenzt Erleichterungen schaffen. Den Angriff auf einen UN-Beobachtungsposten, den jedenfalls zwei Blauhelmsoldaten mit dem Leben bezahlten, bezeichnete der UN-Generalsekretär vor der Presse als "...apparently deliberate targeting ...". 3

Insgesamt beliefen sich nach libanesischen Angaben die Verluste auf 1.184 Tote, darunter 29 Armeeangehörige sowie 4.059 Verletzte einschließlich 63 Armeeangehörige. Nahezu eine Million Binnen-Flüchtlinge konnten erst langsam in ihre Heimatorte zurückkehren, Ende August waren es noch 250.000 Personen. Der Sachschaden wird nach libanesischen Quellen auf rund 3,6 Milliarden Dollar geschätzt.

Nach israelischen Regierungsquellen beliefen sich die eigenen Verluste - vor allem die Opfer der täglich bis zu 150 Raketeneinschläge - auf 55 tote und an die 2.000 verletzte Zivilpersonen. Fast eine halbe Million Zivilisten - etwa die Hälfte der nordisraelischen Bevölkerung - musste evakuiert werden. Im Kampf fielen 118 israelische Soldaten, in Gefangenschaft gerieten zwei. Durch diese Raketen ausgelöste Flächenbrände vernichteten in Nord-Israel etwa 700 Hektar Wald und 1.500 ha Naturlandschaft. Diese Schäden und die übrigen Sachschäden an insgesamt rund 5.500 zivilen Gebäuden beliefen sich nach israelischen Angaben auf ca. 1,1 Mrd. US-Dollar.

\section{Wer ist der Aggressor?}

Aus völkerrechtlicher Sicht stellt sich bei der Beurteilung dieses Konflikts zuerst die Frage, wer als Angreifer zu gelten hat. Dies bestimmt sich nach den vor allem in der UN-Charta enthaltenen Regeln des Friedensrechts, wohingegen die nach dem 13. Juli 2006 folgenden 
bewaffneten Aktionen nach den Maßstäben des humanitären Völkerrechts in Kriegen bzw. bewaffneten Konflikten zu bewerten sind.

Nach den Regelungen des Gewaltverbots ${ }^{4}$ und des Selbstverteidigungsrechts ${ }^{5}$ der UNCharta, konkretisiert durch die Aggressionsdefinition ${ }^{6}$, gilt: "The first use of armed force by a State in contravention of the Charter shall constitute prima facie evidence of an act of aggression..." Im vorliegenden Fall ging die Gewaltanwendung - bei isolierter Betrachtung - von libanesischem Gebiet aus. Es besteht allerdings weitgehend Einigkeit darin, dass der das Selbstverteidigungsrecht auslösende Aggressionsakt von einer gewissen Schwere sein muss. In der völkerrechtlichen Literatur ${ }^{7}$ und in der Rechtsprechung des IGH $^{8}$ werden Scharmützel im Grenzgebiet, auch wenn sie "nur" vergleichsweise wenige Menschenleben kosten, als "Grenzzwischenfall" oder "action short of war" qualifiziert. Allgemein fallen Grenzzwischenfälle als sog. "kleine Gewalt" zwar unter das Gewaltverbot des Art. 2 Abs. 4 UN-Charta, nicht aber unter den Begriff des bewaffneten Angriffs bzw. der Aggression. Das Gewaltverbot schließt also sowohl den bewaffneten Angriff wie auch die "kleine Gewalt" ein. Das Aggressionsverbot ist demnach Teil des Gewaltverbots, erfasst aber nur die mit einer bestimmten Erheblichkeit und Intensität eingesetzte Gewaltanwendung. Das stellt auch die Aggressionsdefinition klar, indem sie festlegt, der UN-Sicherheitsrat könne zu dem Ergebnis gelangen, dass eine bewaffnete Handlung ohne die erforderliche Schwere den Tatbestand der Aggression nicht erfüllt. ${ }^{9} \mathrm{Ob}$ dies auch für Fälle gilt, in denen über Jahre hinweg mit erheblichen Verlusten für beide Seiten Schläge und Gegenschläge aufeinander folgen, bis schließlich gleichsam zu demjenigen Tropfen, der das Fass zum Überlaufen bringt, ist bislang nicht behandelt oder entschieden worden. Nach hier vertretener Auffassung stellt sich die Entführung zweier und die Tötung dreier israelischer Soldaten am 12. Juli als eine weitere Eskalation der vor allem nach dem 26. Mai 2006 einsetzenden Grenzzwischenfälle dar. Dieser bewaffnete Akt der Hisbollah war zweifelsfrei illegal (und die verantwortlichen Personen können und müssen dafür bestraft werden); er erfüllt jedoch von seiner Schwere her nicht den Tatbestand eines bewaffneten Angriffs i.S. des Art. 51 UNCharta, selbst wenn anlässlich der Entführung drei oder - wie vereinzelt berichtet wird sechs israelische Militärangehörige umgekommen wären.

4

5

6
Art. 2 Abs. 4 UN-Charta.

Art. 51 UN-Charta.

UNGA: Res. 3314 (XXIX) vom 14. 12. 1974.

Vgl. u.a. Bothe, Friedenssicherung und Kriegsrecht, in: Vitzthum (Hrsg.), Völkerrecht, 3. Aufl., Rn. 19; Randelzhofer, Art. 51, MN. 20, in: B. Simma (ed.), The Charter of the United Nations (2nd ed., 2002); Doehring, Völkerrecht, 2. Aufl. (2004), Rn. 1037; Schweisfurth, Völkerrecht, 2006, S. 362.

ICJ Reports 1986, Urteil vom 27. 6. 1986, Nicaragua, $\S \$ 191,211,249$; ICJ, Urteil vom 6. 11. 2003, Oil Platforms, $\S \S 51,64$; ICJ, Urteil vom 19. 12. 2005, Congo v. Uganda, $§ \S 131$ et seq.

UNGA: Res. 3314 (XXIX), a.a.O., Art. 2. 
Die Unterscheidung zwischen "kleiner" und "großer" Gewalt ist ungeachtet im einzelnen schwieriger Abgrenzungsfragen wegen der verschiedenen Rechtsfolgen von weitreichender Bedeutung. Allein der mit der notwendigen Gewaltintensität vorgetragene bewaffnete Angriff löst gemäß Art. 51 UN-Charta das Recht auf individuelle und kollektive Selbstverteidigung aus. Eine bewaffnete Handlung unterhalb dieser Schwelle, also z.B. ein Grenzzwischenfall, löst dagegen lediglich "ein Recht auf sofortige und verhältnismäßige Abwehrmaßnahmen aus, nicht jedoch ein umfassendes Selbstverteidigungsrecht". ${ }^{10}$ Die von der UN-Charta für den Tatbestand der Aggression geforderte Schwere hätten demzufolge nach hier vertretener Auffassung weder der seit 2000 andauernde Raketenbeschuss nordisraelischen Gebiets noch das wiederholte Eindringen von bewaffneten HisbollahKräften auf israelisches Territorium, oder die blutige Entführung der beiden israelischen Soldaten, sondern vielmehr erst die von Israel am 13. Juli begonnenen umfangreichen Bombardements gegen den Flughafen und einen südlichen Vorort von Beirut sowie die Verhängung der Seeblockade vor der libanesischen Küste erfüllt. Somit reklamiert Israel zu Unrecht, hier in Selbstverteidigung gehandelt zu haben. ${ }^{11}$

Darüber hinaus müsste nach dem Recht der völkerrechtlichen Verantwortlichkeit ${ }^{12}$ der Hisbollah-Akt der Entführung und Tötung israelischer Soldaten dem Staat Libanon zurechenbar sein. Hisbollah ist zwar als politische und soziale Bewegung seit 2005 im libanesischen Parlament und auch in der Regierung vertreten, ihr militärischer Arm agiert jedoch autonom und außerhalb jeglicher Militärstrukturen der libanesischen Armee. Die bewaffneten Kräfte der Hisbollah verstehen sich vielmehr als private, nichtstaatliche Miliz, die gewissermaßen einen Gegenpol zur libanesischen Armee bildet. Diese - aus europäischer Sicht schwer nachzuvollziehende Situation innerhalb eines Staates - ist nur vor dem Hintergrund der ohnehin fragilen und durch jahrelangen Bürgerkrieg sowie die israelische und auch syrische Besatzung (letztere erst endend mit der - von der Hisbollah bekämpften "Zedern-Revolution" des Frühlings 2005) weiter geschwächten innenpolitischen Kräftekonstellation im Libanon zu verstehen. Jedenfalls haftet ein Staat gegenüber anderen Staaten für das Verhalten seiner eigenen Streitkräfte und, soweit diesen Milizen und Freiwilligenkorps angegliedert sind, auch für solche. ${ }^{13}$ Die Hisbollah-Milizen zählen jedenfalls zu solchen Verbänden nicht.

Die Entführung und Ermordung israelischer Soldaten durch die außerhalb der Strukturen des libanesischen Militärs handelnden Hisbollah-Kräfte wäre demnach dem Staat Libanon nicht zurechenbar. Allerdings hatte noch Ende Mai 2006 der UN-Generalsekretär durch

10

11

12

13

Bothe, Friedenssicherung und Kriegsrecht, a.a.O., Rn. 19.

Vgl. Art. 3 lit. a, b und c Aggressionsdefinition; vgl. Fn. 6.

Vgl. das Kodifikationsergebnis der Völkerrechtskommission zur Staatenverantwortlichkeit als Anlage zu Res. 56/83 der UNGV vom 12. 12. 2001, Art. 4.

Bothe, Friedenssicherung und Kriegsrecht, a.a.O., Rn. 11. 
seinen Sprecher dringend die Umsetzung der Resolution S/Res/1559(2004) ausdrücklich auch dem Libanon gegenüber angemahnt, was immerhin fraglich erscheinen lassen könnte, ob es damit getan war, dass die libanesische Regierung nach Einsetzen der israelischen Reaktion verlauten ließ, von der Planung dieses Akts nichts gewusst und damit nichts zu tun zu haben. ${ }^{14}$ Gleichwohl kann eine Aggression ihrem Wesen nach nur durch aktives, vorsätzliches Handeln eines Staates, nicht aber durch Unterlassen oder durch Fahrlässigkeit begangen werden. Deshalb kann der Auffassung, der Libanon müsse sich als Ergebnis eigener Schwäche die Hisbollah-Aktion vom 13. Juli 2006 völkerrechtlich zurechnen lassen, nicht gefolgt werden. ${ }^{15}$

Auch der Internationale Gerichtshof hält an seiner Auffassung fest, dass im Sinne von Art. 51 UN-Charta ein bewaffneter Angriff gegen einen Staat nur von einem Staat und nicht von nichtstaatlichen Kräften ausgehen kann. ${ }^{16}$ Es gibt keinen Grund, im vorliegenden Fall von diesem Grundsatz abzuweichen. Dafür sprechen auch die Bestimmungen der Prinzipiendeklaration und der Aggressionsdefinition der UNO, die beide das Gewaltverbot und das Selbstverteidigungsrecht der UN-Charta authentisch interpretieren. Zwar soll sich nach der Prinzipiendeklaration jeder Staat der Organisierung, Anstiftung, Unterstützung oder Duldung von gegen das Territorium anderer Staaten gerichteten Bürgerkriegs- oder Terrorakten enthalten, doch setzt die Duldung ("acquiescing") solcher Akte hier die stillschweigende Einwilligung des betreffenden Staates voraus. ${ }^{17}$

Von einer konkludenten Einwilligung der libanesischen Regierung in die Aktivitäten der Hisbollah kann aber nicht die Rede sein. Den Maßstab dafür, wann ein Staat als Angreifer für gewaltsame Handlungen gilt, die von irregulären Einheiten von seinem Gebiet aus gegen einen anderen Staat verübt werden, liefert die Aggressionsdefinition von 1974 ebenfalls. ${ }^{18}$ Danach sind solche Aktivitäten gemeinhin nur dann als indirekte Aggression zu qualifizieren, wenn sie die Schwere der vorher in der Definition genannten Fälle direkter Aggression ${ }^{19}$ erreichen. Überdies muss der Initiatorstaat nach ständiger Rechtsprechung des IGH die effektive, d.h. umfassende, Kontrolle über die von ihm entsandten Banden und

Daily Star (Libanon), 13 July 2006: "Siniora's Cabinet makes clear it had nothing to do with what happened".

So aber S. Weber, Die israelischen Militäraktionen im Libanon und in den besetzten palästinensischen Gebieten 2006 und ihre Vereinbarkeit mit dem Völkerrecht, Archiv des Völkerrechts (AVR), Bd. 44 (2006), S. 464 ff.

16 ICJ, Advisory Opinion on the Legal Consequences of the Construction of a Wall in the Occupied Territory, 9 July 2004, § 139 mit abweichendem Votum Buergenthal; ICJ, Urteil vom 19. 12. 2005, Congo v. Uganda, $§ 141$

UNGA: Res. 2625 (XXV), Prinzip 1, Gewaltverbot, Abs. 9.

18

UNGA: Res. 3314 (XXIX), a.a.O., Art. 3 lit. g; vgl. Fn. 6.

19

A.a.O., Art. 3 lit. a - f. 
Söldner ausüben. ${ }^{20}$ Weder das eine noch das andere Merkmal ist im Verhältnis zwischen dem Libanon und den Hisbollah-Milizen erfüllt. Somit kommt auch aus dieser Sicht der Libanon nicht als Angreiferstaat in Frage.

Israel kann sich auch nicht durch den Hinweis auf ein angeblich seit den Terroranschlägen vom 11. September 2001 verändertes Verständnis in Bezug auf das Selbstverteidigungsrecht der Staaten von der Verantwortlichkeit als Angreiferstaat befreien. So wird etwa angeführt, dass Staaten als Opfer von Terrorakten seitens ausländischer nichtstaatlicher Terrorvereinigungen vom Recht auf Selbstverteidigung Gebrauch machen könnten, weil der Wortlaut des Art. 51 UN-Charta nicht ausdrücklich den Staat als Urheber eines bewaffneten Angriffs benenne, sondern dies offen lasse. Zur Bestätigung dafür wird auf die Resolutionen des Sicherheitsrats 1368 (2001) vom 12. September 2001 und 1373 (2001) vom 28. September 2001 verwiesen, die die Terroranschläge verurteilen und in ihrer Präambel in allgemeiner Form das Selbstverteidigungsrecht erwähnen, ohne den dafür berechtigten Staat für den Fall des 11. September $2001 \mathrm{zu}$ benennen. ${ }^{21}$ Daraus wird - im Vergleich etwa mit dem Verhältnis USA/Afghanistan - abgeleitet, Israel sei berechtigt gewesen, sich zulasten Libanons gegen den Terror der Hisbollah zu verteidigen. ${ }^{22}$

Diese Argumentation vermag jedoch aus mehreren Gründen nicht zu überzeugen:

1. Das Selbstverteidigungsrecht des Art. $51 \mathrm{UN}-\mathrm{Charta}$ in diese Richtung umdeuten zu wollen, geht fehl. Für die Schöpfer der Charta war klar, dass allein Staaten, nicht aber nichtstaatliche Akteure, als Angreifer in Frage kommen. Deshalb setzten sie dies im Text des Art. 51 voraus. An nichtstaatliche Terrorgruppen war 1945 im Übrigen gar nicht zu denken. Das zeigt die Dokumentation über die Entstehung der Charta. ${ }^{23}$

2. Völkerrecht lässt sich nicht nach Bedarf oder Opportunität ändern. Das gilt ganz besonders für grundlegende Normen, die mit universeller Verbindlichkeit zugleich im Gewohnheitsrecht verankert sind. Um solche Grundregeln handelt es sich beim Angriffsverbot wie beim Selbstverteidigungsrecht. Um eine gewohnheitsrechtliche Erweiterung der daran beteiligten Parteien, nämlich durch die nichtstaatlichen Akteure, zu bewirken, bedürfte es

ICJ Reports 1986, Nicaragua, a.a.O., S. 14.ff., §§ 195, 230, 247.

Vgl. u.a. Franck, Terrorism and the Right of Self-Defence, American Journal of International Law (AJIL), Vol. 95 (2001), S. 840; Frowein, Der Terrorismus als Herausforderung des Völkerrechts, Zeitschrift für Ausländisches Öffentliches Recht und Völkerrecht (ZaöRV) Bd. 62 (2002), S. 887; Krajewski, "Krieg gegen Terrorismus" - politische Rhetorik oder neue Konturen des "Kriegsbegriffs" im Völkerrecht?, AVR Bd. 40 (2002), S. 472; Vorsichtiger: Bruha/ Bortfeld, Terrorismus und Selbstverteidigung, Vereinte Nationen (VN) 5/2001, S. 164.

Vgl. Weber (oben Fn 15), a.a.O.; diesbezüglich wohl auch Tomuschat, Der Sommerkrieg des 
wegen der für den Weltfrieden fundamentalen Bedeutung einer länger währenden, nicht nur wenige Jahre umfassenden Übung der Staaten aller Regionen und des Nachweises ihrer dazu gehörigen Rechtsüberzeugung. Eine gewohnheitsrechtliche Änderung des Selbstverteidigungsrechts könnte nur dann erzielt werden, wenn die überwiegende Staatenmehrheit klar erkennbar zustimmen würde und diese Zustimmung nicht nur durch deren Schweigen zu unterstellen wäre. Eine solche weltumspannende Änderung in der Haltung der Staaten zum Selbstverteidigungsrecht hat sich weder nach 2001 noch davor vollzogen. Keinesfalls können die Ausführungen des UN-Sicherheitsrats zum Selbstverteidigungsrecht in den beiden Resolutionen 1368 und 1373 bereits als allgemeine Zustimmung der UN-Mitgliedstaaten zu einer Neuinterpretation des Art. 51 UN-Charta gedeutet werden. Dies spiegelt sich in der unveränderten Rechtsauffassung des IGH zu diesem Gegenstand wider.

3. Diese unveränderte Haltung der großen Mehrheit der internationalen Staatengemeinschaft kann nicht verwundern, wenn man an die möglichen Folgen der von manchen vorgeschlagenen "Anpassung" des Art. 51 an die Erfordernisse des Antiterrorkampfes denkt. Sie bestünden nämlich darin, dass Staaten, die sich stark genug dazu wähnen, unter dem Vorwand des Aufspürens von Terroristen/Terroristinnen letztlich nach Belieben mit ihren Streitkräften - unter Berufung auf das Selbstverteidigungsrecht - in andere Staaten einfallen und bei dieser Gelegenheit gleich eine missliebige Regierung beseitigen könnten. Der Aushebelung des Gewaltverbots und der Wiedereinführung des Rechts des Stärkeren wären auf diese Weise Tür und Tor geöffnet. Daran denkt die Staatenmehrheit offenbar, wenn sie einer derartigen Änderung des bisher konsentierten Konzepts des Rechts auf Selbstverteidigung ihre ausdrückliche Zustimmung verweigert.

Nachdem eine gezielte Aktion der israelischen Armee noch am 12. Juli zur Verfolgung der Entführer auf libanesischem Gebiet erfolglos blieb, wäre es angezeigt gewesen, dass Israel zusammen mit der libanesischen Regierung gemeinsam nach Wegen zur Beilegung der durch die Hisbollah herbeigeführten Eskalation sucht. Stattdessen setzte am 13. Juli die hinsichtlich der Intensität, der Mittel und Methoden der Kriegführung unangemessene fünfwöchige Militäraktion gegen den Libanon ein, die weder zur Befreiung der beiden Geiseln noch zur Auslöschung der Hisbollah geführt hat.

Da die israelische Regierung behauptet, in Wahrnehmung des Selbstverteidigungsrechts gehandelt zu haben, hätte sie im übrigen nach Art. 51 UN-Charta unverzüglich, also noch am 13. Juli, den UN-Sicherheitsrat von ihren Maßnahmen in Kenntnis setzen müssen, was aber nicht erfolgt ist. Israel hat den Rat vor vollendete Tatsachen gestellt und ihm so die Gelegenheit genommen, ggf. weitere Maßnahmen zur Durchsetzung der Resolution 1559 (2004) zum Schutz Israels zu ergreifen. So prüft auch der IGH im Rahmen eines geltend gemachten Selbstverteidigungsrechts, ob der betreffende Staat überhaupt schon gegenüber 
dem Sicherheitsrat Vorgänge geltend gemacht hatte, deretwegen er sich zur Selbstverteidigung genötigt gesehen hat. ${ }^{24}$

\section{Bewertung nach humanitärem Völkerrecht}

Konfliktparteien des am 13. Juli 2006 einsetzenden internationalen bewaffneten Konflikts waren Israel und die Hisbollah. ${ }^{25}$ Die Hisbollah agierte als eine de facto Konfliktpartei, denn sie hatte Südlibanon unter ihrer faktischen (militärischen) Kontrolle. Als derartige Konfliktpartei ist sie an die Regeln des humanitären Völkerrechts gebunden. ${ }^{26}$ Diese ergeben sich vor allem aus den vier Genfer Abkommen (GA) vom 12. 8. 1949 sowie aus der Haager Landkriegsordnung (HLKO) vom 18. 10. 1907. ${ }^{27}$ Beiden Konfliktparteien sind freilich in unterschiedlichem Umfang - Verstöße gegen humanitärrechtliche Regelungen vorzuwerfen. Diese Verstöße beziehen sich in erster Linie auf die Grundregel des Kriegsrechts, dass Kriegshandlungen nur gegen Kombattanten und militärische Objekte zu richten, die Zivilbevölkerung und zivile Objekte aber zu schützen sind. ${ }^{28}$

So ist, trotz der legitimen Absicht, den Raketenbeschuss und den Nachschub der Hisbollah zu unterbinden, die weitreichende Bombardierung von Wohnhäusern und ganzen Wohnblöcken sowie der zivilen Infrastruktur, wichtiger Versorgungseinrichtungen und Energiezentralen durch die israelische Luftwaffe eine offensichtliche Verletzung der einschlägigen Regelungen des humanitären Völkerrechts. ${ }^{29}$ Die Zahl ziviler Personenopfer und die Höhe der materiellen Schäden steht in keinem vertretbaren Verhältnis zu dem jeweils erlangten militärischen Nutzen. So wurde im südlibanesischen Ort Quana, aus dem zuvor über hundert Raketen abgefeuert worden waren, ein dreistöckiges Wohngebäude zerstört. Die meisten Bewohner, darunter auch viele Kinder, kamen dabei ums Leben, andere blieben vermisst. $^{30}$ In solchen Fällen und anders als bei der Zerstörung des Hisbollah-Senders "Al

ICJ, Urteil vom 19. 12. 2005, Congo v. Uganda, a.a.O., § 145:" The Court would first observe that in August and early September 1998 Uganda did not report to the Security Council events that it had regarded as requiring it to act in self-defence."

Hisbollah kann hier als ein de facto Gebilde i.S. des Kriegsrechts betrachtet werden, das an das geltende Kriegsrecht gebunden ist.

Vgl. Bothe, Als Rechtsbegriff hat der "Krieg" ausgedient, Interview in der Süddeutschen Zeitung, 30.08.2006.

27 Israel hat die Zusatzprotokolle zu den Genfer Abkommen vom 8. 6. 1977 nicht ratifiziert.

28

29

30

Vgl. insb. Art. 27ff. GA IV.

Vgl. z.B. Art. 25 HLKO.

Erklärung des UN-Sicherheitsrats S/PRST/2006/35 vom 30.07.2006; vgl. auch BBC News, 31 July 2006: "Human Rights Watch accused the Israeli Defence Forces (IDF) of treating southern Lebanon as a "free-fire zone". It said the failure to distinguish between civilians and combatants 
Manar", liegt die Unverhältnismäßigkeit offen auf der Hand, so dass der Verweis auf "Kollateralschäden" als Rechtfertigung ungeeignet ist. Als Kollateralschäden wären die Zerstörungen vor allem nicht einzustufen, wenn Israel die vielen zivilen Opfer und Schäden bewusst in Kauf genommen hätte, wie es immerhin eine Äußerung des israelischen Generalstabschefs, Chalutz, nahelegen könnte, "die Uhr im Libanon um 20 Jahre zurückzudrehen", falls die entführten Soldaten nicht freigelassen würden. ${ }^{31}$

Freilich muss man berücksichtigen, dass - auch nach UN-Feststellungen - die Hisbollah ihre Raketenstellungen vielfach inmitten von Wohngebieten postiert hatte. Damit verstieß die Hisbollah ganz offensichtlich und schwerwiegend gegen die grundlegende FairnessRegel des geltendes Kriegsvölkerrechts zur Trennung militärischer und ziviler Ziele. Dennoch war die israelische Armee nicht völlig befreit von jeder Beachtung des Proportionalitätsprinzips. Sie durfte vor allem nicht ganze Wohngebiete attackieren. Das gilt erst recht etwa für Wohnhäuser in Gegenden, von denen aus Katjuscharaketen israelisches Territorium gar nicht erreichen konnten. So wurden in und um Beirut Wohnquartiere mit der Begründung bombardiert, es residierten dort überwiegend Anhänger bzw. Führungskader der Hisbollah. Die mehrfachen Ankündigungen von Bombardements und Warnungen an die Zivilbevölkerung mit der Aufforderung, sich in Sicherheit zu bringen, vermögen Israel kaum zu entlasten, weil - aus unterschiedlichen Gründen, darunter auch Verlassensverbote der Hisbollah - nicht alle betroffenen Personen fliehen konnten und die nachfolgende Zerstörung der Wohnungen und anderer ziviler Objekte nach Kriegsrecht dennoch gegen Israel zu Buche schlägt.

So kann man wohl angesichts der schwerwiegenden Verstöße gegen das Verhältnismäßigkeitsprinzip durch Israel im Verlaufe des Libanonkrieges insgesamt zu dem Ergebnis gelangen, dass, selbst dann, wenn man unterstellen würde, Israel habe in Ausübung des Selbstverteidigungsrechts gehandelt, ein Selbstverteidigungsexzess vorläge, der hinsichtlich der Folgen-Bewertung einer Aggression gleichkäme. ${ }^{32}$

Als schwere Verletzung des humanitären Völkerrechts gelten auch Missachtung und Missbrauch von Schutzzeichen, etwa des Roten Kreuzes bzw. Roten Halbmondes sowie der Vereinten Nationen. ${ }^{33} \mathrm{Ob}$ sich Berichte bewahrheiten, nach denen es zu solchen Verstößen beidseits gekommen sein soll, müssen die eingeleiteten Untersuchungen zeigen. Gleiches

could be judged as a war crime, and called for an UN probe into the conflict. Israel has insisted that Hezbollah sheltered in Qana and used it as a base to fire rockets across the border..." 2006: "Israel authorizes 'severe' response to abductions". 
gilt für den Untersuchungsbericht zu den Einzelheiten darüber, wie es am 25.07.2006 zu dem israelischen Angriff auf den UN-Beobachterposten gekommen ist.

Der Abwurf von Streubomben über libanesischem Gebiet wird israelischerseits damit gerechtfertigt, dass die geltenden humanitärrechtlichen Verträge bislang kein ausdrückliches Verbot dieser weltweit in vielen Arsenalen vorgehaltenen Waffen enthielten. Auch wenn es in der Tat ein ausdrückliches Verbot nicht gibt, so handelt es sich doch um verbotene Waffen im Sinne der HLKO, weil ihr Gebrauch geeignet ist, unnötige Leiden zu verursachen. $^{34}$

Auch die Hisbollah soll Streubomben eingesetzt haben. ${ }^{35}$ Sie muss sich darüber hinaus anlasten lassen, durch die Positionierung ihrer Raketenstellungen inmitten von libanesischen Wohnsiedlungen die dort ansässigen Zivilpersonen faktisch in Geiselhaft für ihre militärischen Aktionen genommen zu haben, was das humanitäre Kriegsrecht ebenfalls ausdrücklich verbietet. ${ }^{36}$

\section{Völkerrechtliche Verantwortlichkeit}

Nach hier vertretener Auffassung muss für die Verletzung des Aggressionsverbots der Staat Israel verantwortlich gemacht werden. Zuständig für diese Feststellung wäre primär der UN-Sicherheitsrat. Daran ist aber vor allem wegen des Vetorechts der USA derzeit nicht zu denken. Die Diktion der Resolution 1701(2006) lässt dagegen eher erkennen, dass sich die im Rat vertretenen Staaten um des lieben Friedens willen dem Druck der USA gebeugt und Formulierungen zugestimmt haben, die eine spätere Verurteilung Israels möglichst verhindern sollen. Umgekehrt steht aber auch eine Klage Libanons vor dem IGH augenscheinlich nicht in Rede. Nach geltendem Völkerrecht träfe Israel die Pflicht zur umfassenden Wiedergutmachung des von ihm verursachten Schadens. ${ }^{37}$ Demnach hätte Israel die dem Staat Libanon entstandenen Schäden zu ersetzen. Angesichts der Dimension der dort angerichteten Zerstörungen hat die UNO ihre Mitgliedstaaten zur Unterstützung des Libanon aufgerufen. Dabei darf jedoch nicht in Vergessenheit geraten, dass die Hauptverantwortung für den Wiederaufbau beim Verursacherstaat verbleiben muss.

Die völkerstrafrechtliche Verantwortlichkeit der politischen und militärischen Führung für die Auslösung einer Aggression bleibt davon prinzipiell unberührt. Jedoch fehlt hierfür zur

34

35

36

37

Vgl. Art. 23 lit. e HLKO.

So die Recherchen von Human Rights Watch. Vgl. Berliner Zeitung vom 20. 10. 2006, S. 7.

Vgl. dazu Art. 34 GA IV.

Vgl. Art. 34 Entwurf der Völkerrechtskommission der UNO zur Staatenverantwortlichkeit. Official Records of the General Assembly, 56 Session, Suppl. No. 10 (A/56/10), chp. IV, E 1. 
Zeit ebenfalls das geeignete Forum, zumal der Internationale Strafgerichtshof von Israel und Libanon nicht anerkannt wird und noch nicht über den konkreten Tatbestand der individuellen Verantwortlichkeit für Aggressionen verfügt. ${ }^{38}$ Bei den oben erwähnten jeweiligen Verstößen gegen das humanitäre Völkerrecht dürfte es sich aufgrund ihrer Tragweite zumeist nicht um einfache Vergehen, sondern um Kriegsverbrechen i.S. der Genfer Abkommen $^{39}$ und des Art. 8 des Römischen Statuts des Internationalen Strafgerichtshofs ${ }^{40}$ handeln. Dafür hätten sich die politischen und militärischen Verantwortlichen sowohl Israels als auch der Hisbollah zu verantworten. Dies könnte vor nationalen oder vor internationalen Gerichten geschehen. Die Aussichten stehen aber dafür momentan ebenfalls nicht gut. Allerdings zeigt die jüngere Geschichte, dass jähe Wendungen nicht ausgeschlossen sind: Als Milosevic seine Verbrechen begangen hatte, hat er es gewiss nicht für möglich gehalten, dass er einige Zeit später - neben anderen - vor dem Jugoslawientribunal stehen würde.

\section{Ansätze zur Lösung des Konflikts}

Es wäre naiv zu glauben, dass die vom UN-Sicherheitsrat beschlossene Aufstockung des im Libanon stationierten Truppenkontingents auf 15.000 Soldaten und deren Ausstattung mit einem robusten Mandat die Lösung des hier behandelten Konflikts wäre. Diese Maßnahme könnte bestenfalls für einen bestimmten Zeitraum günstigere Rahmenbedingungen für eine politische Lösung schaffen, nicht mehr. Eine politische Lösung kann aber nur dann erfolgreich erzielt werden, wenn der Konflikt zwischen Israel und dem Libanon bzw. jeweils beider mit der Hisbollah in die komplexe Nahostproblematik eingeordnet wird, in deren Mittelpunkt das Palästinaproblem steht, und zu der u.a. auch die israelisch/syrische Golan-Frage und die Annexion Ost-Jerusalems gehören. Die Gewaltspirale kann nur durchbrochen und Frieden im Nahen Osten erreicht werden, wenn alle Beteiligten grundlegend umdenken und umsteuern. Das verlangt den Verzicht auf den Vorrang des Militärischen und die konsequente Hinwendung zu den friedlichen (diplomatischen) Mitteln der Beilegung der angehäuften Streitigkeiten. Ein solcher Prozess sollte von vertrauensbildenden Maßnahmen begleitet werden. 41

Dies setzt in erster Linie voraus, dass einerseits das Existenzrecht Israels nicht weiterhin in Frage gestellt wird, andererseits Israel nicht länger die Bildung eines palästinensischen Staates behindert und die seit 1967 anhaltende Besatzung der Westbank beendet, wo die

38

39

40

41

Dazu Werle, Völkerstrafrecht, 2003, S. 445 ff.

Vgl. Art. 147 GA IV, Art. 130 GA III, Art. 50 GA I.

Vgl. BGBl. 2000, II, S. 1394.

Vgl. Johannsen, Waffenruhe im Libanon: Ruhe vor dem nächsten Sturm?, Hamburger Informationen zur Friedensforschung und Sicherheitspolitik, Ausgabe 39/2006, S. 9 f. 
Palästinenser Repressionen und Demütigungen ausgesetzt sind. Dass Gewalt Gegengewalt hervorruft, lehrt die Geschichte, insbesondere auch diejenige des Staates Israel seit 1948. Dabei war Israel anfangs infolge der Bedrohung durch die arabischen Nachbarn überwiegend in der Opferrolle und benötigte die Unterstützung von außen. Inzwischen ist Israel nicht nur wegen seines Kernwaffenpotentials - eine weit überlegene und gefürchtete Militärmacht in der Region. Das rigorose Vorgehen gegen die Palästinenser und die oft unverhältnismäßigen Einsätze seiner Streitkräfte wurden in der Vergangenheit jedenfalls von der UN-Generalversammlung den auf beiden Seiten zu unterlassenden "acts of terrorism" zugeordnet. $^{42}$

Bei den noch nicht im vertraglichen Frieden mit Israel lebenden arabischen Nachbarn hat sich eine Gefühlsmischung aus Demütigung, Ohnmacht und Hass angestaut, die nur von beiden Seiten wieder abgebaut werden könnte. Israel, wiederholt angegriffen, beruft sich zwar zutreffend auf sein Recht auf sichere Grenzen und Achtung seiner territorialen Integrität, doch gilt dieser Grundsatz auch, sobald es (Stichworte Golan, Westbank und OstJerusalem) um das gleiche Recht von Nachbarn geht. So hat der IGH z.B. festgestellt, dass auch der Bau der Mauer zwischen Israel und der Westbank auf dem Gebiet des künftigen palästinensischen Staates völkerrechtswidrig ist. ${ }^{43}$ Israel reklamierte in der Vergangenheit oft für sich einen Sonderstatus und machte unter Berufung auf die eigene Sicherheit Rechte geltend, die ihm nach der UN-Charta nicht zustehen. ${ }^{44}$

Die deutsche Bundesregierung hat sich im Verlaufe des Libanonkonflikts nach anfänglichem Zögern zunehmend an die israelisch-amerikanische Linie angelehnt. Sie unterließ es in der frühen Phase des Konflikts, einen sofortigen Waffenstillstand zu fordern, wie er von vielen, auch vom UN-Generalsekretär, wenngleich erst später unter dem Eindruck der Tragödie in Quana, gefordert wurde. Sie verzichtete auf jegliche Kritik an der israelischen Kriegsführung und ließ an ihrer Verbundenheit mit Israel keinen Zweifel. ${ }^{45}$

"...Taking note also of the position of the Palestine Liberation Organization which condemns all acts of terrorism, whether committed by States or individuals, including acts of terrorism committed by Israel against the Palestinian people and the Arab nation..." (A/Res/40/96, 12.12.1985); "....3. Condemns all acts of violence, including all acts of terror, provocation, incitement and destruction, especially the excessive use of force by Israeli forces against Palestinian civilians, resulting in extensive loss of life, vast numbers of injuries and massive destruction..." (A/Res/ 58/99, 09.12.1999); "....condemning all acts of violence and terror against civilians on both sides, including the suicide bombings and extrajudicial executions..." (A/Res/58/21, 22.01.2004).

ICJ, Legal Consequences of the Construction of a Wall in the Occupied Palestinian Territory, 9 July 2004, § 142 .

Vgl. Seidel, Die Palästinafrage und das Völkerrecht, AVR, Bd. 44 (2006), S. 138 ff.

45

So stimmte Deutschland am 11.08. 2006 im neuen UN-Menschenrechtsrat mit dessen übrigen EU-Mitgliedern gegen eine mit 27 zu 11 Stimmen bei 8 Enthaltungen angenommene Resolution, in der allein Israel wegen gravierender Verletzung der Menschenrechte im Libanonkonflikt verurteilt wurde. Vgl. United Nations. General Assembly, A/HRC/S-2/2, p. 3, 17 August 2006. 
Darin dürfte sich ein generelles Problem deutscher Nahost-Politik offenbaren: Aus der moralischen Verantwortung, die die Deutschen aus der Vergangenheit ohne jeden Zweifel für die Juden haben, wird wohl der Schluss gezogen, die Bundesrepublik müsse sich jeder Kritik an der Politik des Staates Israel enthalten. Aus Furcht vor dem möglichen Vorwurf des Antisemitismus versagt sich dann die deutsche Politik, Israel bei Verstößen gegen das Völkerrecht zu rügen. ${ }^{46}$ Damit weicht sie nicht nur von den Prinzipien ihrer eigenen, auf allgemeine Friedenserhaltung und Wahrung der Menschenrechte gerichteten Politik ab, sondern sie begibt sich u.U. auch der Chance, ungeachtet der EU-Mitgliedschaft im so genannten "Nahost-Quartett" als unparteilicher Vermittler zwischen den Konfliktparteien im Nahen Osten anerkannt zu werden.

Stellt sich aber für Deutschland die Frage nicht vielmehr so, dass es aus der erwähnten historischen Verantwortung gerade die Verpflichtung hat, seine Stimme deutlich gegenüber jedem zu erheben, der das völkerrechtliche Friedensgebot verletzt und schwere Menschenrechtsverletzungen begeht, auch wenn es Israel sein sollte. Der bekannte Soziologe und Politikwissenschaftler Alfred Grosser, der 1933 wegen seiner jüdischen Herkunft nach Frankreich emigrieren musste, äußerte sich in einem Interview dahingehend, es sei die israelische Politik selbst, die den Antisemitismus fördere. ${ }^{47}$

Wenn die Krise in den Beziehungen zwischen Israel und Libanon Teil des großen Nahostkonflikts ist, dann böte sich eine Gesamtlösung auf einer Konferenz an, um zugleich Ruhe an allen Fronten zu schaffen. Dies dürfte aber wegen der Komplexität und Schwierigkeiten der Probleme sowie wegen der Unterschiedlichkeit der beteiligten Staaten kaum erreichbar sein. So dürften die Verhandlungen je nach Reifegrad nacheinander anzugehen sein, so wie es bisher auch zu den Friedensregelungen mit Ägypten und Jordanien gekommen ist, die das Existenzrecht Israels nicht mehr in Frage stellen. Zur Schaffung einer Atmosphäre des Vertrauens in den bilateralen Beziehungen zwischen Israel und Libanon kommt es libanesischerseits vor allem darauf an, die internen Machtverhältnisse zu stabilisieren und den militärischen Einfluss Syriens wie der Hisbollah zurückzudrängen. Die internationale Staatengemeinschaft hat mit der Bereitstellung von Finanzmitteln auf der Geberkonferenz vom Januar 2007 einen ersten wichtigen Beitrag zum Wiederaufbau des Landes geleistet. Sie unterstützt den Libanon anhaltend dadurch, dass sie - darunter auch Deutschland - in

Lediglich die Entwicklungshilfeministerin, H. Wieczorek-Zeul, hatte in den ersten Wochen des Libanon-Krieges einen sofortigen Waffenstillstand verlangt und später allein Israel wegen des Einsatzes von Streubomben kritisiert. Die Präsidentin des Zentralrats der Juden in Deutschland warf ihr deshalb einen antijüdischen Kurs vor und verlangte ihren Rücktritt. Vgl. Berliner Zeitung vom 30.08. 2006, S. 4.

47

Interview in der Berliner Zeitung vom 15. 08. 2006: "....Kritik an Israel und Antisemitismus haben nichts miteinander zu tun. Es ist vielmehr Israels Politik, die den Antisemitismus in der Welt fördert. Natürlich gibt es einen latenten Antisemitismus sowohl auf der extrem rechten als auch auf der extrem linken Seite, der dadurch belebt wird...". 
Verwirklichung der UN-Resolution 1701 (2006) mit militärischen Mitteln gegen die illegale Lieferung von Waffen jedenfalls in den Süd-Libanon vorgeht. Um trotz schmerzlicher Rückschläge ${ }^{48}$ den Weg zum Verhandlungstisch zu ebnen, ist es Aufgabe aller Beteiligten, auf jedwede Gewalt zu verzichten sowie Zurückhaltung und Toleranz zu üben.

So im Libanon z.B. die Attentate vom Juni 2007. Dazu der Sprecher des UN-Generalsekretärs: "...The Secretary-General was saddened by the news of today's terrorist attack in Beirut that killed MP Walid Eido, his son and six others, while injuring ten more people. He condemns this attack in the strongest possible terms as a heinous crime aimed at destabilizing Lebanon" (Statement vom 13. Juni 2007). "...The Secretary-General is deeply saddened by and condemns in the strongest possible terms the terrorist attack on a United Nations patrol in South Lebanon yesterday that killed six and injured two other United Nations peacekeepers belonging to the Spanish contingent....The Secretary-General notes the fragility of the situation in Lebanon and reiterates the importance of UNIFIL's mandate for stability in the area...." (Statement vom 25. Juni 2007). 